\title{
Efficient Power Allocation for Video over Superposition Coding
}

\author{
Chun Pong Lau, Kamran Jamshaid, and Basem Shihada \\ Computer, Electrical and Mathematical Science and Engineering Division \\ King Abdullah University of Science and Technology \\ Thuwal 23955-6900, Saudi Arabia \\ \{lau.pong, kamran.jamshaid, basem.shihada\}@kaust.edu.sa
}

\begin{abstract}
In this paper we consider a wireless multimedia system by mapping scalable video coded (SVC) bitstream upon superposition coded (SPC) signals, referred to as (SVC-SPC) architecture. Empirical experiments using a software-defined radio (SDR) emulator are conducted to gain a better understanding of its efficiency, specifically, the impact of the received signal due to different power allocation ratios. Our experimental results show that to maintain high video quality, the power allocated to the base layer should be approximately four times higher than the power allocated to the enhancement layer.
\end{abstract}

\section{INTRODUCTION}

The popularity of smart mobile handheld devices has increased the number of users who access the Internet through wireless connections [1]. Web and e-mail services are no longer the largest bandwidth consumers in Internet. Instead, most of the traffic is driven by multimedia-related services. Multimedia Broadcast Services (MBS) defined under the leading-edge industry standards (e.g., Worldwide Interoperability Microwave Access (WiMAX) and Long Term Evolution (LTE)) support broadcast of scalable coded video bitstreams using single modulated broadcasting signal.

Due to multiple receivers with wide diversity of wireless channels, it is a challenge to select a proper transmission rate (or modulation scheme) for achieving the desired service. This is also referred to as multi-user channel diversity problem [2]. The difference of Signal to Noise Ratio (SNR) among receivers is affected by distance as well as interference with other wireless devices. Multi-paths fading attenuates the received signal power. For certain base station coverage area, signal power for users located at different positions varies. Therefore, different modulation schemes are implemented to support different data transmission rates.

With multi-user channel diversity, a dilemma exists in the selection of transmission rates. By taking an aggressive modulation scheme at the transmitter saves resource blocks while sacrificing the users with bad SNRs (e.g., at the cell edge); but by taking a conservative modulation scheme, more users can successfully demodulate the received signal at the expense of more resource block consumption [3]. Besides multi-user channel diversity, short-term channel fluctuation serves as another impairment on the wireless multicast system. This is particularly a problem with successively refinable bitstreams under SVC, in which the loss of base layer data results in loss of the entire video frame even if the corresponding enhancement layer data is successfully received and decoded.

The SVC-SPC joint coding architecture is an interesting approach to resolve the aforementioned issues. It has been shown that SPC multicast for scalable bitstreams can fully explore the channel capacity in presence of channel fluctuation and multi-user channel diversity by superimposing multiple quality layers into a single SPC modulated signal [2]. With the SVC-SPC architecture, the signals launched in the air, also referred to as Superposition Coded Multicast (SCM) signals, can avoid the situation where the received enhancement layer bits are not useful in contributing to the video quality due to the loss of the corresponding bits of base layer. This can greatly improve system throughput even in the presence of channel errors. At the same time, the multi-user channel diversity problem can be effectively mitigated since the receivers with poor channels can decode and obtain the base layer data for a basic video perceptual quality, while receivers with good channel conditions can obtain, in addition to the base layer, the higher quality layers to refine the data from lower layers for improved video quality.

The main contribution of this paper lies in empirical evaluation of the SVC-SPC architecture using NI PXI 5641R along with CAASP software module that emulates the IEEE 802.16e MAC protocol. The paper examines the impact on the throughput performance due to changing power allocation ratios of the SCM signals at transmission.

The rest of the paper is organized as follows. Section II introduces the background of this work. A discussion on power allocation is presented in Section III. Section IV shows the system architecture and experimental setup. Experimental results are presented and analyzed in Section V. The paper is concluded in Section VI.

\section{BACKGROUND}

In this section we provide a brief overview on scalable video coding and superposition coding.

\section{A. Scalable Video Coding}

In layered transmission scheme, SVC is employed because of its adaptability. It is the Annex $G$ extension of the H.264/AVC video compression standard, which is one of the most commonly used video codec formats [4]. The 
SVC video bitstream consists of single base layer and one or more enhancement layers. Base layer contains the minimal information of the video and the enhancement layers contain additional information which can further improve the quality beyond the base layer. The base layer bitstream is backward compatible with H.264/AVC which can be decoded and played independently without other enhancement layers of the original bitstream with less quality. Therefore, any additional enhancement layers being decoded and played contributes to better video quality.

\section{B. Superposition Coding}

Superposition coding is a physical-layer technique enabled by a hardware circuitry that allows a transmitter to send individual information to multiple receivers within a single wireless broadcast signal [5], [6]. SPC signals contain multiresolution modulated symbols, enabling a receiver to decode its own information as well as its peers' assuming a good channel condition. As proposed in [7], a two layers SCM signal is used for broadcasting scalable video instead of using one modulation for each transmission. In SCM, each broadcasting signal is superimposed by a base quality layer of video bitstreams data modulated by a lower-order modulation scheme such as BPSK, and an enhancement quality layer of video bitstreams by a higher-order modulation scheme such as 16QAM. Using SCM, a single superimposed signal can serve different channel conditions within a base station coverage area. Users with good channel can decode the full quality video while users with bad channel can only decode the base layer.

\section{Power AllocAtion}

Power allocation between the two layers in SCM is an important factor for increasing the coverage area and maintaining high video quality. Incorrect power allocation between SPC layers can dramatically deteriorate video reception. For example, assume an SPC with two layers, base and enhancement, superimposed into one broadcasting signal. The superimposed signal $x$ can be taken as the summation of the two vectors expressed in terms of the corresponding amplitudes and phases in a constellation diagram. This is formed by the conventional approach using $m_{1}$-QAM and $m_{2}$-QAM, where $m_{2}>m_{1}$. Note that BPSK is treated by a 2-QAM modulation scheme for the generalization purpose. The resultant constellation diagram of signal $x$ consists of $m_{1} m_{2}$ points, each with a unique amplitude-phase combination determined by the allocation ratio of energies for the $m_{1}$-QAM and $m_{2}$-QAM signals, denoted by $E_{1}$ and $E_{2}$, respectively. The overall energy is constant and similar to using generic multicasting. The total transmitted energy between both layers has the following relationship,

$$
\begin{gathered}
E=E_{1}+E_{2} \\
E_{1}=\beta E \\
E_{2}=(1-\beta) E
\end{gathered}
$$

where $E$ is the total energy and $\beta$ is defined as the power allocation ratio for the base layer of total energy. A larger $\beta$ value allocates more energy to base layer [8].

In order to provide better understanding of the system, we provide the following power allocation case study. Assume that there exists three power allocation ratios, including $\beta=$ $0.5,0.7992$ and 0.9091 . The three ratios show the extreme and appropriate cases of power allocation. For illustration, we plot some example constellation diagrams of a SPC signal (BPSK + QPSK) in Figure 1 with their corresponding $\beta$ values. When $\beta=0.5$, both base and enhancement layer are allocated with equal energy. Base layer value is decoded first according to the in-phase value. However, the constellation points in the middle of the map are very close to the center value 0 which may be wrongly decoded at the receiver demodulator. When $\beta=0.9091$, most of the power is allocated to base layer. The crosses are far away from each other to reduce the error rate in base layer under high attenuation. However, the constellation points of the enhancement layer are close to each other, making it difficult to determine the correct symbol when channel becomes noisy. $\beta=0.7992$ shows a balance power allocation between both layers which is the best ratio among different $\beta$ values.

Figure 2 shows the constellation diagrams from the receiver when attenuation is $9 \mathrm{~dB}$. As mentioned earlier, when $\beta=$ 0.5 , the constellation points in the middle are too close to each other, leading to a symbol error rate of more than $5 \%$. Thus, the receiver cannot extract the correct symbols from base layer. If using this ratio for transmission, users cannot correctly receive the video when attenuation is not high. When $\beta=0.9091$, two groups of points can be distinguished, which indicates that the base layer symbol error rate is less than $1 \%$. However, it is difficult to further determine the original position in the enhancement layer, leading to a high symbol error rate of greater than $80 \%$ in enhancement layer. If using this ratio, users with high attenuation can receive the broadcast with base layer quality but users in middle attenuation will lose their enhancement layer data. When $\beta=0.7992$, both base layer and enhancement layer can also be decoded with a low symbol error rate of less than $1 \%$. Therefore, users with medium attenuation can also obtain the enhancement quality video instead of only base layer as in the prior case.

We conclude that it is important to find a suitable value of $\beta$ to maintain a high quality transmission. In the next section, we show that the power allocated to the base layer should be approximately four times higher than the power allocated to the enhancement layer to maintain high video quality for majority of users.

\section{Testbed Setup}

In this section, we introduce our SVC-SPC implementation. An emulation testbed is developed following a cross-layer design. The main functioning models are the Application layer (APP), the Medium Access Control layer (MAC) and the Physical layer (PHY). 

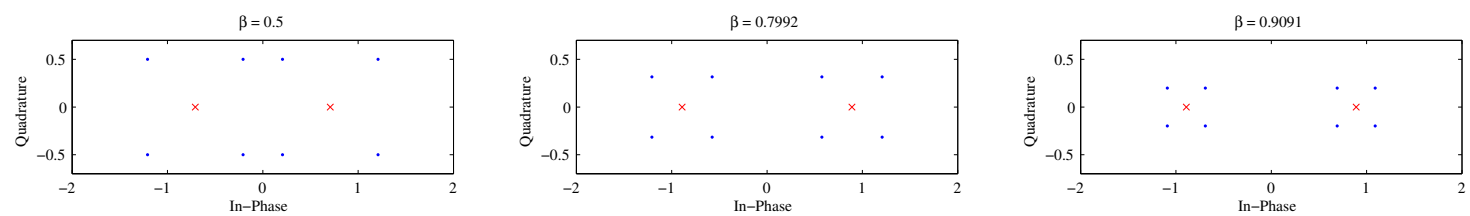

Fig. 1: Constellation of SPC with different power allocation ratios $\beta$. (Circle points are the constellation being transmitted and crosses are the reference location of the base layer which are not actually used)
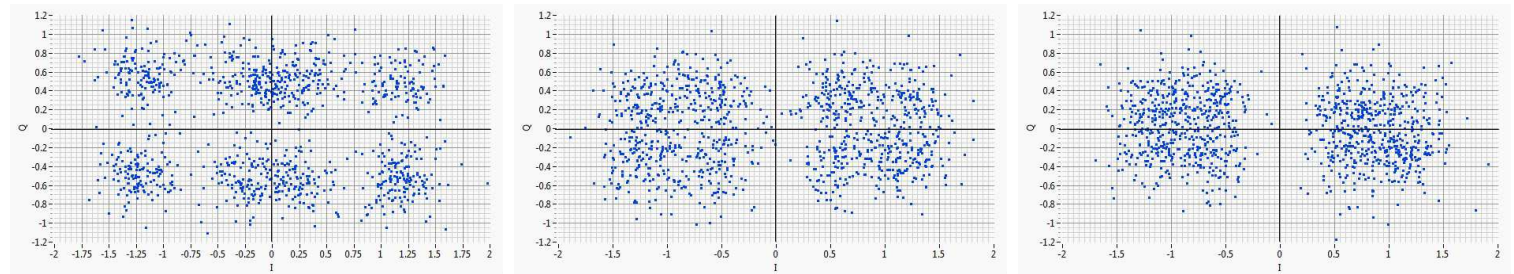

Fig. 2: Constellation screenshots at the receiver with attenuation of 9dB. (each point represents a received symbol)(left: $\beta=0.5$, middle: $\beta=0.7992$, right: $\beta=0.9091$ )

\section{A. Application Layer}

Prior to transmission, scalable video content is encoded with two quality layers. In the emulation testbed, the application layer is implemented in a PC. The transmitter PC is responsible for preparing the video content and extracting the two video quality layers into two separate buffers of the MAC layer in the emulator machine. The receiver PC acts as a user mobile device to receive the video from MAC layer and combines the two quality layers into one video file for playback.

\section{B. Medium Access Control Layer}

The MAC layer consists of two sub-layers, MAC Core and MAC Convergence Sub-layer (MAC CS). The two layers communicate using real-time FIFOs queue. MAC Core provides several capabilities including system access, PHY configuration, bandwidth allocation, and connection maintenance. MAC Convergence sub-layer mainly performs the check or insertion of CRCs, check or insertion of data headers and insertion or stripping of data padding. It also provides transformation or mapping of data exchanged between the MAC Cores.

\section{Physical Layer}

The PHY layer of the transmitter emulator includes the following blocks: Service Access Point (SAP) Interface, Forward Error Correction (FEC) unit, and OFDMA Processor. The receiver PHY layers contains the same blocks along with a synchronization unit. SAP interface is the main Tx interface between the MAC and PHY layers in both transmitter and receiver. At the transmitter, FEC unit implements the channel encoding algorithms which is used to encode the payload and send the coded data to the OFDMA processor. OFDMA processor receives the coded payload from the FEC unit, permutes the user sub-channels according to the fixed PUSC scheme, modulates the OFDM signals, and then sends the processed samples to the Digital to Analog Converter. At the receiver, the synchronization unit is responsible for frame synchronization (i.e., Carrier Frequency Offset (CFO), Time
Offset (TO) estimation, compensation, storage, and retrieval of the estimated CFO and TO values). The input of this unit is the I/Q signals from the Analog to Digital converter. CFO and TO values are initially estimated from the input signals by exploiting the frame preamble; then their estimates are updated for every OFDM symbol. The compensated I/Q signals are then sent to the OFDMA Processor. The OFDMA processor demodulates the OFDM signals, recovers the user sub-channels and then sends the processed samples to the FEC unit in order to recover the transmitted payload. FEC unit implements the channel decoding algorithm using the Viterbi decoder to extract the payload and send the recovered data to SAP interface.

\section{Testbed Layout}

Since our testbed consists of single transmitter and single receiver, several experimental attempts are repeated with the same settings at different attenuation values to emulate different users under various channel conditions for the same broadcasting signal coverage.

Two emulators named CAASP-Tx and CAASP-Rx act as the transmitting base station and the receiving user device as shown in Figure 3. These emulators are implemented by National Instruments PXIe-1062Q chassis, with embedded controller PXIe-8130. IF transceiver PXIe-5641R, RF Up converter PXI-5610 for transmitter and RF Down converter PXI-5600 for receiver. Both emulators are connected by two coaxial RF cable with a variable attenuator acting as the air channel with a predefined signal attenuation. The model of the two RF cables are CBL-3FT-SMSM+ and CBL-6FT-SMSM+ from Mini-Circuits with length $3 \mathrm{ft}$ and $6 \mathrm{ft}$. The insertion loss of the cables is $1.0 \mathrm{~dB}$ and $2.0 \mathrm{~dB}$ respectively when the signal is in the range of $2.5-6 \mathrm{GHz}$. The model of variable attenuation is $4674-30 \mathrm{FS}$ manufactured by ARRA. Although we could have used antennas between the two emulators, using a closed circuit with an attenuator ensures the repeatability of our experiment under all conditions and prevents interference from 


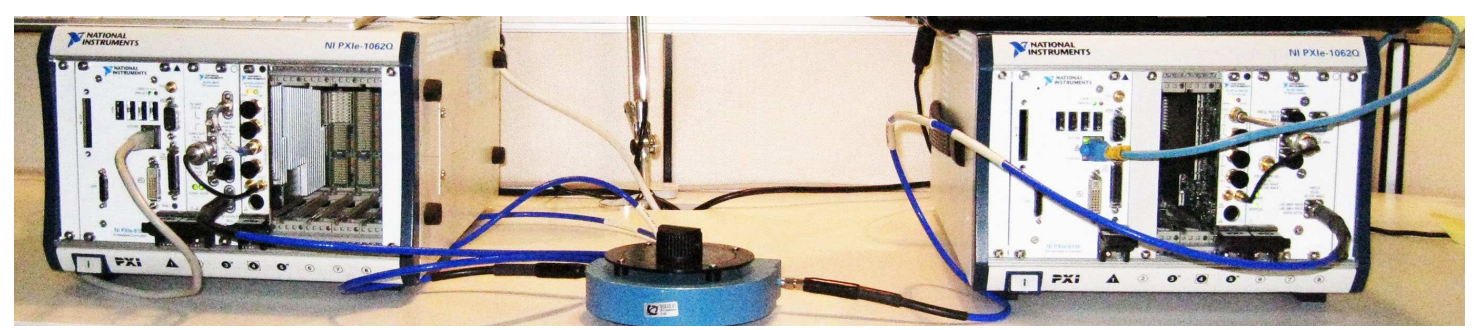

Fig. 3: Emulation testbed. Left side acts as transmitter and right side acts as receiver. The device in the middle is the variable attenuator to manually adjust the channel attenuation

TABLE I: Network Characteristic

\begin{tabular}{|l|c|c|}
\hline Transmission type & SCM & GM \\
\hline Air Interface & IEEE 802.16e-2005 DL Only \\
\hline Duplex mode & \multicolumn{2}{|c|}{ TDD } \\
\hline FFT Size & \multicolumn{2}{|c|}{$1024 \mathrm{Bytes}$} \\
\hline Channel width & \multicolumn{2}{|c|}{$10 \mathrm{MHz}$} \\
\hline Cyclic Prefix & \multicolumn{2}{|c|}{$1 / 4$} \\
\hline Frame length & \multicolumn{2}{|c|}{$5 \mathrm{~ms}$} \\
\hline B. Layer Modulation & BPSK $1 / 2$ & $16 \mathrm{QAM} 1 / 2$ \\
\hline B. Layer Capacity & $821 \mathrm{kbps}$ & $3200 \mathrm{kbps}$ \\
\hline E. Layer Modulation & QPSK $1 / 2$ & N/A \\
\hline E. Layer Capacity & $1641 \mathrm{kbps}$ & $\mathrm{N} / \mathrm{A}$ \\
\hline Radio Frequency & \multicolumn{2}{|c|}{$2.51 \mathrm{GHz}$} \\
\hline Tx Power & \multicolumn{2}{|c|}{$0 \mathrm{dBm}$} \\
\hline Rx Power Sensitivity & \multicolumn{2}{|c|}{$0 \mathrm{dBm}$} \\
\hline
\end{tabular}

the surrounding wireless signals. During a given experiments, the channel attenuation values are kept constant. Table I shows the detailed system characteristics.

1) Average Symbol Energy in SCM: Power allocation ratio $\beta$ cannot be directly configured in the system, instead the power boosting value can be changed from $-12 \mathrm{~dB}$ to $+12 \mathrm{~dB}$ stepped by one $\mathrm{dB}$ for controlling the power on base and enhancement layer separately. Therefore, we perform a simple calculation to transform the modulation and boosting into power allocation ratio.

In generic multicasting (GM), the coordination of each symbol in the constellation diagram are located at the odd integer multiple of the abscissa and ordinate. Since the average symbol energy varies between modulations, it is necessary to be normalized. A scaling factor is applied to QAM in order to normalize the average symbol energy to one. In the generic QAM, symbols are distributed evenly with equal distances in a 2D plane. The average symbol energy of $m$-QAM is given by,

$$
\begin{aligned}
E_{M-\mathrm{QAM}} & =\frac{2 \frac{\sqrt{M}}{2}}{\frac{M}{4}} \sum_{m=1}^{\frac{\sqrt{M}}{2}}[2 m-1]^{2} \\
& =\frac{2}{3}(M-1)
\end{aligned}
$$

Consider the average symbol energy of 16QAM is $\frac{2}{3}(16-$ $1)=10$, and the scaling factor is $\frac{1}{\sqrt{10}}$. Each of the symbol alphabets in both abscissa and ordinate coordination are multiplied by the scaling factor to normalize the overall average
TABLE II: Average Symbol Energy and Power Allocation Ratio

\begin{tabular}{|c|c|c|c|}
\hline $\begin{array}{c}\text { Power } \\
\text { Allocation } \\
\text { Ratio } \beta\end{array}$ & $\begin{array}{c}\text { Base } \\
\text { Boosting } \\
(\mathrm{dB})\end{array}$ & $\begin{array}{c}\text { Enhance } \\
\text { Boosting } \\
(\mathrm{dB})\end{array}$ & $\begin{array}{c}\text { Average Symbol } \\
\text { Energy after } \\
\text { boosting } C_{\text {boost }}\end{array}$ \\
\hline$\beta=0.9091$ & -1 & -11 & 0.8738 \\
\hline$\beta=0.8632$ & -1 & -9 & 0.9202 \\
\hline$\beta=0.7992$ & -1 & -7 & 0.9939 \\
\hline$\beta=0.7153$ & -1 & -5 & 1.1106 \\
\hline$\beta=0.6131$ & -2 & -4 & 1.0291 \\
\hline$\beta=0.5$ & -3 & -3 & 1.0024 \\
\hline
\end{tabular}

symbol energy to one. For example, the coordination of the top left symbol of 64QAM before scaling is $(-7,7)$ in I/Q plane. After the multiplication with the scaling factor $\frac{1}{\sqrt{42}}$, the new coordination is $(-1.0801,1.0801)$. The location of symbols is also determined by the power boosting parameter in the system by the following equation,

$$
C_{\text {boost }}=C \times 10^{\left(\frac{b}{20}\right)}
$$

where $C_{\text {boost }}$ is the new coordination after boosting, $C$ is the original coordination after normalization, $b$ is the boosting value range from $-12 \mathrm{~dB}$ to $+12 \mathrm{~dB}$ with step size $1 \mathrm{~dB}$.

A similar process is used for the enhancement layer. Since the step sizes of boosting value are fixed to one, it introduces a situation where not every combination of boosting values can maintain the average symbol energy after superposition to one. Table II shows a set of combinations that the average symbol energy is close to one with corresponding power allocation ratio $\beta$. This set of combinations will be evaluated in the following sections.

2) Video Characteristics: The broadcast video is a movie trailer of 'Sintel' developed by Durian Open Movie Project with standard definition resolution (480p), encoded in H.264/SVC format by JSVM 9.18 [9], [10]. It consists of 2 scalable layers in quality scalability, base layer with Quantization Parameters (QP) 35, and enhancement layer with QP 25 . The group of picture structured as IBPB, with key picture encoded for every I- and P-frames. Table III shows the details of video parameters.

\section{EXPERIMENTAL RESUlTS}

We collected 920 data samples for our analysis. The samples are collected with different network and video parameters as 
TABLE III: Video Properties and Correlated Modulations

\begin{tabular}{|l|c|c|}
\hline Number of Frames & \multicolumn{2}{|c|}{1242 frames } \\
\hline Frame Rate & \multicolumn{2}{|c|}{$30 \mathrm{fps}$} \\
\hline Resolution & \multicolumn{2}{|c|}{$864 \times 480$ pixels } \\
\hline Group of Pictures & \multicolumn{2}{|c|}{2} \\
\hline Intra Period & \multicolumn{2}{|c|}{ CAVLC } \\
\hline Symbol mode & \multicolumn{2}{|c|}{ Yes } \\
\hline Inter-layer Prediction & \multicolumn{2}{|c|}{ Yes } \\
\hline Encoding Key Picture & Base & Enhance \\
\hline Layer & 35 & 25 \\
\hline QP & $521.63 \mathrm{kbps}$ & $1686.88 \mathrm{kbps}$ \\
\hline Bitrate & $56.01 \mathrm{~dB}$ & $59.12 \mathrm{~dB}$ \\
\hline Encoding PSNR & BPSK 1/2 & QPSK 1/2 \\
\hline Corr. SCM Modulation & \multicolumn{2}{|c|}{$16 \mathrm{QAM} 1 / 2$} \\
\hline Corr. GM Modulation & \multicolumn{2}{|c|}{}
\end{tabular}

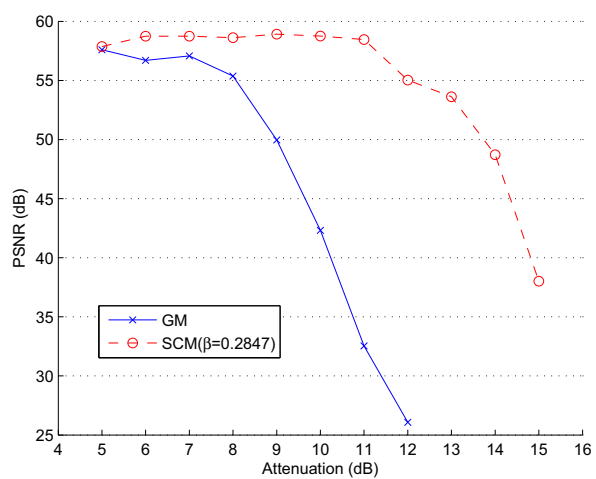

Fig. 4: PSNR Comparison between GM and SCM

described in the previous section. Each sample was emulated as broadcasting the video in 40 seconds. In our experiments, we used Peak Signal-to-Noise Ratio (PSNR) as video quality assessment metric. It is a full-reference objective video quality metric and scales to indicate the error introduced by video compression and network transmission. A higher value of PSNR represents a higher video quality at the receiver.

\section{A. $G M$ vs SCM}

In traditional method, GM can only broadcast identical signal to all users within the base station coverage area. Since the broadcast video is in high quality with a high bitrate $2207.5 \mathrm{kbps}$, an aggressive modulations scheme (16QAM) is used. Consider three users with attenuation values of $6 \mathrm{~dB}$, $10 \mathrm{~dB}$ and $14 \mathrm{~dB}$. From Figure 4, user 1 at $6 \mathrm{~dB}$ can receive the video with almost highest quality (PSNR). However, user 2 at $10 \mathrm{~dB}$ received a significant quality drop. The last user at $14 \mathrm{~dB}$ even could not receive the broadcast correctly. By employing SCM, a superposition-coded signal was broadcasted from the base station. SCM provides a good video quality to both user 1 and user 2 at $6 \mathrm{~dB}$ and $10 \mathrm{~dB}$. User 3 at $14 \mathrm{~dB}$ can also receive acceptable quality of video from decoding base layer information without enhancement layer data. The screenshots in Figure 5 show the video quality at $10 \mathrm{~dB}$ transmitted by GM and SCM $(\beta=0.7153)$ and $14 \mathrm{~dB}$ by SCM $(\beta=0.7153)$. We notice more distortion in the picture by GM under the same channel condition.

\section{B. SCM Power Allocation Evaluation}

In order to further enhance the performance of SCM, a better power allocation ratio has to be carefully chosen as found in Sections III. During this experiment, several power allocation ratios are compared. Assume each power allocation ratio has similar average energy per symbol, the difference between them is the allocated power ratio $\beta$ between base and enhancement layer. A higher value of $\beta$ implies more energy allocated to base layer and less energy allocated to the enhancement layer.

The results in Figure 6 show that when the channel condition is good, $(5-6 \mathrm{~dB})$, each ratio performs similarly and deliver high video quality with lower Packet Error and Loss Rate (PELR). When channel worsens (7-10dB), for an allocation ratio with lower $\beta$ (i.e., $\beta=0.5$ ) the video quality degraded faster than other ratios. This is because less energy is allocated to base layer (i.e., most of the important information stored in base layer was not correctly received, although PELR of enhancement layer (E-PELR) was not high). The allocation ratios with the higher $\beta$ (i.e., $\beta=0.9091$ ) performed better compared to $\beta=0.5$. However, the PSNR begins to drop at $7 \mathrm{~dB}$ and remains constant until reaching $15 \mathrm{~dB}$, since E-PELR is increasing with a low PELR in base layer (i.e., more energy was allocated to base layer). The best value for this experiment is $\beta=0.7992$ which shows that when video quality started decreasing at $11 \mathrm{~dB}$, with increasing E-PELR, more users in medium channel condition (7-11dB) can receive a higher video quality than other ratios. On the other hand, base layer PELR (B-PELR) was kept in a lower value until the channel gets worse (16dB).

\section{Effects of PELR in Enhancement Layer}

When E-PELR increases, it degrades the video quality in terms of PSNR due to packet loss. In Figure 6a, almost every line from the PSNR suddenly dropped in the middle attenuation $(7-12 \mathrm{~dB})$ and later increases to reach PSNR 55.8dB at high attenuation (13-14dB). We observe that the PSNR of $\beta=0.7992$ (stars) at $12 \mathrm{~dB}$ and $\beta=0.8632$ (squares) at $10 \mathrm{~dB}$. These two points dropped to $55.0 \mathrm{~dB}$ and $53.9 \mathrm{~dB}$ respectively, then increase to $55.8 \mathrm{~dB}$ and $55.7 \mathrm{~dB}$ at attenuation $13 \mathrm{~dB}$. In other words, the PSNR drops below the base layer encoding PSNR (56.0dB) when E-PELR was high but less than 100\%, the PSNR increases to base layer encoding PSNR when EPELR was equal to $100 \%$. The reason of this behavior is the existence of corrupted or lost packet in enhancement layer which affect the decoding process. When E-PELR is less than $100 \%$, some of the corrupted enhancement layer packets are used to decode the video, degrading its quality. If all of the enhancement packets are lost, only the base layer packets were received and correctly decoded, it can reach the maximum PSNR of the base layer. We conclude that if the corrupted enhancement layer packets are discarded on the fly, it can increase the PSNR of video up to the encoding PSNR of base layer. The ideal scenario should be a direct step function in Figure $6 \mathrm{c}$ jumps from $0 \%$ to $100 \%$ to prevent any corrupted enhancement layer packets affect decoding process. 

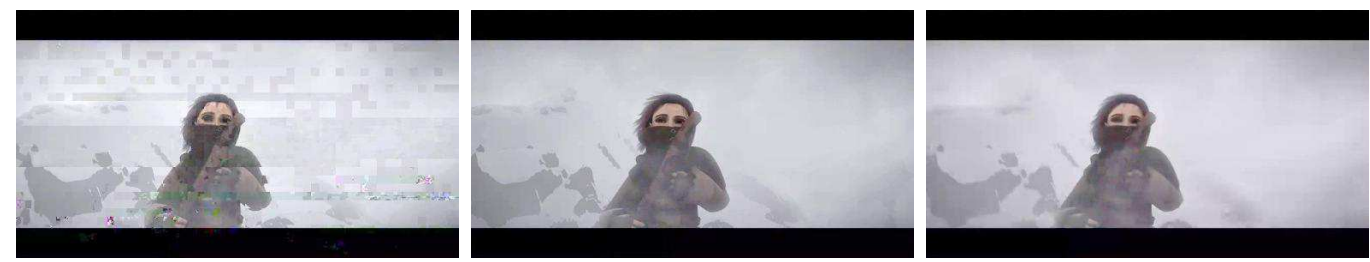

Fig. 5: Screenshots of transmitted video, left: GM at 10dB (Average PSNR: $42.3 \mathrm{~dB}$ ), middle: SCM ( $\beta=0.7153)$ at $10 \mathrm{~dB}$ (Average PSNR: $58.8 \mathrm{~dB})$, right: $\operatorname{SCM}(\beta=0.7153)$ at $14 \mathrm{~dB}$ (Average PSNR: $48.7 \mathrm{~dB}$ )

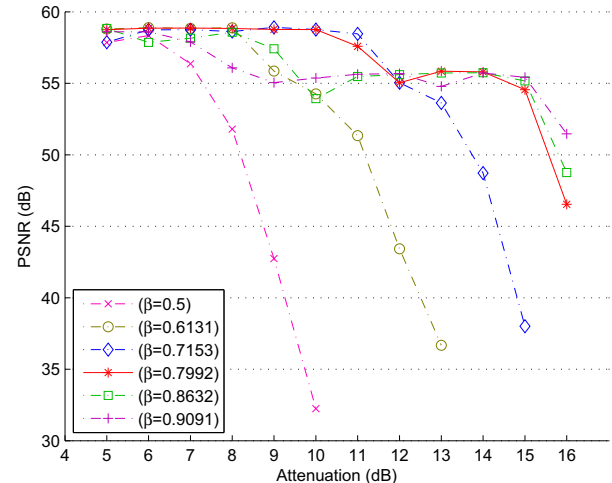

(a) PSNR

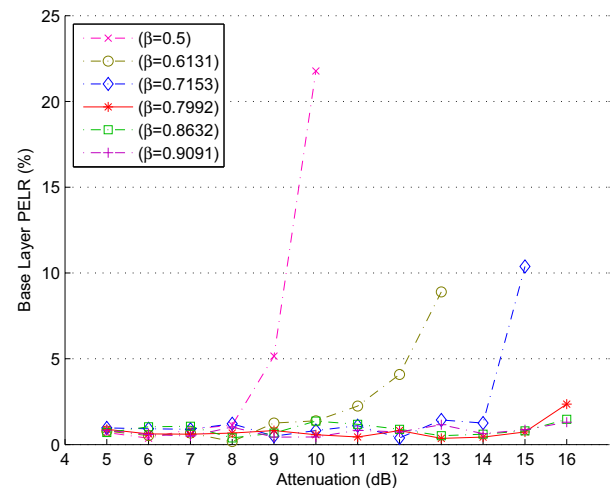

(b) Base Layer PELR

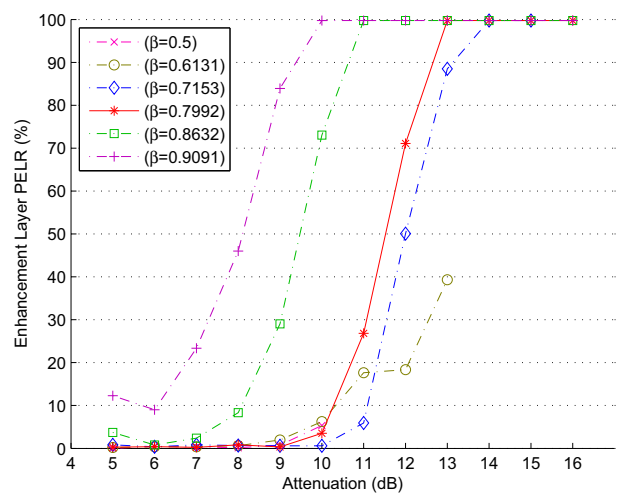

(c) Enhancement Layer PELR

Fig. 6: PSNR, base layer PELR and enhancement layer PELR comparison

\section{CONCLUSION}

This paper empirically demonstrates that SCM can efficiently overcome the multiuser channel diversity problem with an appropriate selection of power allocation ratio between base and enhancement layers. Researchers and system operators should take special consideration for selecting the appropriate power allocation ratio while deploying SPC multicasting scheme. We showed that the power allocated to the base layer should be approximately four times higher than the power allocated to the enhancement layer. We also found that lost or corrupted enhancement layer packets can significantly affect the video quality. Discarding corrupted or lost enhancement layer packets is essential to maintain good video quality.

\section{REFERENCES}

[1] T. Chen, "30th anniversary of the PC and the post-PC era," Network, IEEE, vol. 25, no. 5, pp. 2-3, 2011. [Online]. Available: $\mathrm{http}: / /$ ieeexplore.ieee.org/xpls/abs_all.jsp?arnumber $=6033028$

[2] J. She, F. Hou, P.-H. Ho, and L.-L. Xie, "IPTV over WiMAX: Key Success Factors, Challenges, and Solutions [Advances in Mobile Multimedia]," IEEE Communications Magazine, vol. 45, no. 8, pp. 87-93, 2007. [Online]. Available: http://ieeexplore.ieee.org/xpl/freeabs_all.jsp?arnumber $=4290319$

[3] C. Tarhini and T. Chahed, "On capacity of OFDMA-based IEEE802. 16 WiMAX including Adaptive Modulation and Coding (AMC) and inter-cell interference," in ... LANMAN 2007. 15th IEEE Workshop on, 2007, pp. 139-144. [Online]. Available: http://ieeexplore.ieee.org/xpls/abs_all.jsp?arnumber=4295989

[4] H. Schwarz, D. Marpe, and T. Wiegand, "Overview of the Scalable Video Coding Extension of the H.264/AVC Standard," IEEE Transactions on Circuits and Systems for Video Technology, vol. 17, no. 9, pp. 1103-1120, Sep. 2007. [Online]. Available: http://ieeexplore.ieee.org/lpdocs/epic03/wrapper.htm?arnumber=4317636

[5] T. Cover, "Broadcast channels," Information Theory, IEEE Transactions on, vol. 18, no. 1, pp. 2-14, Jan. 1972. [Online]. Available: http://ieeexplore.ieee.org/lpdocs/epic03/wrapper.htm?arnumber=1054727

[6] S. Bopping and J. M. Shea, "Superposition coding in the downlink of CDMA cellular systems," in Wireless Communications and Networking Conference, 2006. WCNC 2006. IEEE, vol. 4. IEEE, 2006, pp. 19781983.

[7] J. She, X. Yu, F. Hou, P.-H. Ho, and E.-H. Yang, "A Framework of Cross-Layer Superposition Coded Multicast for Robust IPTV Services over WiMAX," 2008 IEEE Wireless Communications and Networking Conference, pp. 3139-3144, 2008. [Online]. Available: http://ieeexplore.ieee.org/lpdocs/epic03/wrapper.htm?arnumber=4489579

[8] J. C.-C. Ho, "Logical superposition coded modulation for wireless video multicasting," Ph.D. dissertation, Univ. of Waterloo, Waterloo, Canada, 2009. [Online]. Available: http://www.uwspace.uwaterloo.ca/handle/10012/4323

[9] T. Roosendaal, "Sintel," in ACM SIGGRAPH 2011 Computer Animation Festival, ser. SIGGRAPH '11. New York, NY, USA: ACM, 2011, p. 71. [Online]. Available: http://doi.acm.org/10.1145/2019001.2019066

[10] T. Wiegand, G. J. Sullivan, J. Reichel, H. Schwarz, and M. Wien, "Joint scalable video model JSVM-9," Joint Video Team, Doc. JVT- V, 2007. 